\title{
Canoes of the Grand Ocean coordonné par Anne Di Piazza et Erik Pearthree
}

\section{Gilles Bounoure}

\section{OpenEdition}

1 Journals

\section{Édition électronique}

URL : http://journals.openedition.org/jso/6169

DOI : $10.4000 /$ jso.6169

ISSN : 1760-7256

\section{Éditeur}

Société des océanistes

\section{Édition imprimée}

Date de publication : 15 décembre 2010

Pagination : $271-273$

ISBN : 978-2-85430-027-7

ISSN : 0300-953x

\section{Référence électronique}

Gilles Bounoure, "Canoes of the Grand Ocean coordonné par Anne Di Piazza et Erik Pearthree », Journal de la Société des Océanistes [En ligne], 130-131 | 2010, mis en ligne le 15 décembre 2010, consulté le 24 septembre 2020. URL : http://journals.openedition.org/jso/6169; DOI : https://doi.org/10.4000/jso. 6169 
Il s'agit plutôt d'une récitation de nom de clans et de lignages et de leurs alliances et parcours.

Ces chants de toutes sortes sont un autre moyen de la tradition orale kanak pour conserver et transmettre ce qui marque la vie quotidienne. Tristes ou joyeux, ils sont l'empreinte de l'histoire et de la vie, mais aussi parfois l'expression de l'espoir d'un monde meilleur.

Isabelle LEBLIC, CNRS-LACITO, Villejuif

Géo 370 : dossier Nouvelle-Calédonie, décembre 2009, pp. $46-107$.

Le magazine Géo a publié en décembre dernier un dossier spécial de 60 pages sur la Nouvelle-Calédonie. Une interview de Christian Karembeu, célèbre footballeur français originaire des îles Loyauté, introduit une série d'articles abordant d'importants sujets d'actualité de la Nouvelle-Calédonie : la protection du lagon d'Ouvéa inscrit en 2008 au patrimoine mondial de l'UNESCO (pp. 54-59) avec un très court texte commentant de belles photos de cette île réputée « la plus proche du paradis », une carte présentant les six zones de l'archipel inscrites à ce même patrimoine avec leurs espèces remarquables (pp. 60-61), « les avancées chaotiques du destin commun » (pp. 70-79), « des squats dans la ville » (pp. 80-82), « Nickel, le bon filon enfin partagé » (pp. 84-88), « ainsi parlent les ancêtres » (p. 90), un encart dépliant sur une collection privée d'art kanak accompagnant un article sur le collectionneur William Costes (p. 97), « des artistes qui assurent la relève » (pp. 98-100), pour terminer par la présentation d'un sentier de randonnée entre eau et forêt dans le sud de la Grande Terre (pp. 102-104) et quelques conseils avant de partir (p. 106).

Très grand public, ce dossier peut constituer une introduction à la Nouvelle-Calédonie d'aujourd'hui mais laisse sur sa faim tout connaisseur du pays. De même, les orientations bibliographiques données sont des plus limitées. À lire pour le plaisir des belles photos plus que pour le contenu des textes.

Isabelle LEBLIC, CNRS-LACITO, Villejuif

Di Piazza Anne and Erik Pearthree (eds), 2008. Canoes of the Grand Ocean, Oxford, Archaeopress, BAR International Series 1802, vI-154 p., bibliogr., cartes, nombreuses ill. dans le texte.

En présentant cette réunion de huit contributions d'archéologie maritime, A. Di Piazza souligne d'emblée ce qu'a de plaisant et de fascinant le vieux terme français de « Grand Océan » choisi pour intituler ce volume. Dans toute fascination entre forcément une part d'appréhension, qu'on songe à celle des anciens navigateurs défiant les vastitudes et les colères du Pacifique ou à celle des scientifiques d'aujourd'hui qui tentent de reconstituer leur histoire, sujet immense et d'une importance essentielle pour la compréhension du peuplement de l'Océanie. Sur ce thème réellement fascinant et avec l'aspect plaisant que lui donnent ses nombreuses illustrations, cet ouvrage ajoute à cette part nécessaire d'appréhension une autre des plus évitables et superflues, avec sa fragile couverture à « dos collé » qui laisse vite échapper son contenu en feuilles volantes et finit par rendre sa lecture hasardeuse. Les bibliothèques devront le faire relier avant de le mettre à la disposition du public, sous les alizés comme sous d'autres vents.

Les contributions sont réparties en trois sections (un peu abusivement dénommées « sessions » comme s'il s'agissait d'un colloque) à dominante respectivement historique, technique et spéculative, non sans incursions nécessaires des auteurs d'un domaine à l'autre, l'architecture navale venant ainsi fournir des arguments aux historiens, comme on va le voir. L'ambition de la première partie, «Navigators as the principal actors in cross-cultural encounters », est d'éclairer par leur contexte historique les témoignages des premiers Européens à avoir décrit les navigateurs traditionnels du «Grand Océan». Anne Salmond («Voyaging exchanges: Tahitian Pilots and European Navigators ", pp. 23-46) revient notamment sur la « carte de Tupa'ia » dressée par Cook sur les indications du pilote tahitien, mais elle en compare aussi les données avec ce que retirèrent des connaissances des insulaires les deux vaisseaux de guerre espagnols de passage en 1774, pour dresser en conclusion une liste d'îles remarquable de précision. Spécialiste chevronné de l'auteur de l'Essai sur la construction navale des peuples extraeuropéens... (1843), Eric Rieth («Traditional Oceanic Canoes as seen by Admiral Paris », pp. 47-68) montre sur quel travail d'observation directe, mais aussi de documentation historique repose cette œuvre classique. Par contraste avec les représentations européennes, Richard Feinberg poursuit son analyse des conceptions traditionnelles des insulaires d'Anuta, et les examine ici dans leurs implications maritimes (« Polynesian Representations of Geographical and Cosmological Space: Anuta, Solomon Islands», pp. 69-84).

Due à Carlos Mondragón et Miquel Luque Talaván, la contribution ouvrant cette première partie (« Early European Descriptions of Oceanic Watercraft. Iberian Sources and Contexts », pp. 9-22) est peut-être la plus ambitieuse et la plus sujette à discussion. Elle revient sur deux escales des navigateurs espagnols commandés successivement par Mendaña et Quirós, la première au sud-ouest de Santa Isabel en février 1568, la deuxième à Taumako en avril 1606. Dans le premier cas, les insulaires de Furona étant venus proposer un porc aux Espagnols en échange d'un canot que ces derniers leur avaient pris, les auteurs de l'article n'ont pas de mal à en inférer que les insulaires ne prenaient pas les Blancs pour des fantômes ou des esprits. Dans le deuxième cas que les auteurs examinent plus longuement (pp. 13-20), les Espagnols restèrent à terre une dizaine de jours et rapportèrent des descriptions admiratives des embarcations des insulaires. Or ces témoignages ne correspondraient pas au célèbre te puke propre à Taumako, où cherche à le remettre en usage l'association « Vaka 
Taumako Project » initiée en 1993 par David Lewis et Marianne George, mais plutôt à des kalia ou à des tongiaki venus de Tonga, et semblables à ceux que dépeignirent peu après Schouten et Le Maire en 1616, puis Gilsemans et Tasman en 1643.

La thèse semble à première vue séduisante et de nature à corroborer tout ce qu'on a pu écrire sur «l'empire maritime tongien » de la première moitié du deuxième millénaire de notre ère. On pourrait ajouter qu'il y eut certainement jadis des relations maritimes réciproques entre Tonga et Taumako puisque Gerd Koch, enquêtant en 1966 sur place, s'y était fait montrer «la direction de Tonga » par son grand ami Basil Tevake de Nifiloli, qu'il présentait comme « le dernier 'capitaine' » traditionnel de haute mer en Polynésie (Koch, 1971 : 153-154). Tevake fut deux ans plus tard l'informateur privilégié de David Lewis (1994, p. 87 et passim) et après sa mort, la référence principale du "Vaka Taumako Project» dont C. Mondragón et M. L. Talaván ont repris une large part de la bibliographie (note 17 p. 14), incomplète notamment des travaux de G. Koch.

On doit pourtant à ce savant une description très détaillée (ibid. : 144-153) du petit te puke (7,07 mètres) qu'il a rapporté au musée de Berlin, et il n'aurait pas été inutile de la mettre en regard des indications plus vagues des journaux de navigation de Quirós et de ses compagnons. Les plus précises concernent les dimensions de ces embarcations qui auraient été longues de 16,70 mètres environ, " quite different from those of a Taumako te puke ", concluent les deux auteurs (p. 19). Mais à propos des te puke, J. Neyret (1976a : 82-83) était fondé à avancer (d'après Hornell) que « ces pirogues étaient beaucoup plus grandes autrefois ». Walter Coote (1886:107, texte ici conforme à l'édition anglaise de 1883) a vu à Nufili des te puke qui « avaient environ 40 pieds de long ». Selon Koch (1971 : 149), leur longueur traditionnelle était de « 14 mètres en moyenne » et Marianne George ellemême, dans une adresse aux " supporteurs du 'Vaka Taumako Project' » datée du 6 juillet 1997, écrivait que «la longueur minimale d'un vrai tepuke est de 12 mètres, même si certains anciens se souviennent de tepuke pouvant atteindre 20 mètres "). Quant aux tongiaki, ce type d'embarcation tongienne pouvait dépasser 30 mètres (Koch, 1985 : 19) et devait « atteindre couramment de 20 à $25 \mathrm{~m}$ de long " (Neyret, 1976b : p. 115). C'était aussi la dimension moyenne des lakatoi des Mailu parcourant les côtes sud-est de la Nouvelle-Guinée (voir par exemple Helfrich, $2005: 41)$.

Même savamment vérifiée par les auteurs d'après les étalons en usage dans l'Espagne de l'époque (pp. 17-19), la longueur prêtée à l'embarcation ne saurait permettre à elle seule de distinguer entre voiliers hauturiers de Tonga et de Taumako. Il aurait certainement fallu scruter certains autres détails d'architecture navale relevés par les Espagnols à Taumako, notamment le recours au bambou (caña) pour les lisses et le «mât» (mast, mastil, selon le texte en version anglaise et espagnole reproduit p. 17). Or, rappelait G. Koch (1985: 25), si les éléments de « mâture » sont généralement en bois de fer (Casua- rina) en Polynésie, le recours au bambou est typique de la Micronésie (et certainement aussi des écarts polynésiens à influences micronésiennes comme Taumako). Du reste, le témoin cité par C. Mondragón et M. L. Talaván, Diego de Prado y Tovar, qui suivrait quelques mois plus tard avec Luis Váez de Torres une route différente de celle de Quirós, a-t-il eu réellement l'occasion d'examiner de près une de ces embarcations gréées et en état de manœuvrer?

Selon Quirós (ou son secrétaire Belmonte qu'il remanie), c'est dans les hangars à bateaux de Taumako (« des maisons plus grandes, qui abritent des embarcations... », Quirós, 2001 : 242, voir aussi Baert, 1999 : 305) que les Espagnols purent observer en détail ces voiliers, probablement "désarmés » comme il était habituel en ces lieux. Rejeter l'idée qu'il s'agissait là d'un type ancien du te puke local (pour autant que les descriptions espagnoles soient exactes), hypothèse la plus vraisemblable, reviendrait à admettre que les Tongiens ou d'autres avaient installé à Taumako une base navale complète de son arsenal édifié à terre, un Diego Suarez ou un Okinawa avant la lettre, ce dont les visiteurs espagnols ne se seraient pas aperçus durant les dix jours qu'ils y passèrent en avril 1606. Telles sont pourtant, explicites ou non, les suppositions conduisant les auteurs à "l'importante conclusion » que Taumako aurait été l'un des carrefours historiques (« crossroads», p. 13) des navigateurs du sud-ouest du «Grand Océan », nouvelle conjecture qu'ils n'étayent d'aucune prise en compte des intérêts respectifs qui auraient fait éventuellement des uns, à Taumako, les hôtes plus ou moins complaisants des autres, venus d'ailleurs.

Les quatre articles formant les deux dernières « sessions » du volume sont largement plus techniques et s'adressent avant tout aux spécialistes de la construction navale (A. Horridge, "Origins and relationships of Pacific canoes and rigs », pp. 85-105; R. Veccella, «Dugout and sewn plank canoe construction on Raivavae, Austral Islands ", pp. 107-120) et à ceux de la navigation, de la météorologie marine et de leur modélisation informatique (C. Avis, A. Montenegro, A. Weaver, "Simulating island discovery during the Lapita expansion », pp. 121-142; B. M. Evans, «Simulating Polynesian double-hulled canoe voyaging, combining digital and experimental data to prepare for a voyage to Rapa Nui [Easter Island] », pp. 143-154). Puisque les embarcations de Raivavae furent longtemps «très semblables à celles de Rurutu », on complétera les descriptions de R. Veccella par les témoignages anciens soigneusement rassemblés par P. Vérin (1969 : 206-211) sur les pirogues «cousues » de Rurutu. A. Horrige s'excuse (p. 85 n. 1) de ne pouvoir livrer qu'un exposé «bref et touffu » de l'évolution des embarcations océaniennes, et de ce qu'elle doit aux apports techniques extérieurs, indonésiens d'abord puis européens à partir du XVI ${ }^{\mathrm{e}}$ siècle (p. 98), mais son article est déjà de grand intérêt, notamment $\mathrm{du}$ fait de son ancienne et profonde connaissance des marines du Sud-Est asiatique. Illustrant les progrès accomplis récemment à la fois dans la modélisation informatique et dans la compréhension de la navigation traditionnelle dans le 
Pacifique, avec l'introduction de paramètres aujourd'hui mieux pris en compte et étudiés, les variations climatiques en premier lieu, les deux dernières contributions viennent aussi rappeler que le «Grand Océan » fait appel à presque toutes les ressources du savoir humain, autre élément qui le rend fascinant, aujourd'hui comme jadis.

\section{RÉFÉRENCES CITÉES}

BAERT Annie, 1999. Le Paradis Terrestre, un mythe espagnol en Océanie. Les voyages de Mendaña et de Quirós, 1567-1606, Paris, L'Harmattan.

Coote Walter, 1886. L'océan Pacifique occidental, description des groupes d'îles au nord et à l'est du continent australien, Paris, Librairie Charles Delagrave.

Helfrich Klaus, 1985. Boote aus Melanesien und Australien, in Gerd Koch (herausg.), Boote aus aller Welt, Berlin, Museum für Völkerkunde, pp. 33-54.
Koch Gerd, 1971. Materielle Kultur der Santa CruzInseln, unter besonderer Berücksichtigung der RiffInseln, Berlin, Museum für Völkerkunde.

-, 1985. Boote aus Polynesien und Mikronesien, in Gerd Koch (herausg.), Boote aus aller Welt, Berlin, Museum für Völkerkunde, pp. 11-32.

LEWIS David, 1994. We, the navigators, The Ancient Art of Landfinding in the Pacific, $2^{\mathrm{d}}$ edition, Honolulu, University of Hawai'i Press.

Neyret Jean, 1976a et b. Pirogues océaniennes, tome I et tome II, deuxième édition, Paris, Association des Amis des musées de la Marine.

Quirós Pedro Fernández (de), 2001. Histoire de la découverte des régions australes, traduction et notes d'Annie Baert, Paris, L'Harmattan.

VÉRIN Pierre, 1969. L'ancienne civilisation de Rurutu (îles Australes, Polynésie française). La période classique, Paris, ORSTOM. 